\title{
Chemistry in Space
}

$\mathrm{T}$ HE chief impression left by the first meeting of Section B (Chemistry) of the British Association at York on Sept. 1, was the soundness of the foundations on which stereochemistry has been built. After thirty years of revolution in our ideas of the nature of the physical world, the basic conceptions of chemistry need little modification and their assimilation into the body of the new atomic physics only gives them a clearer meaning and a wider usefulness.

This is well exemplified by the first of the two main themes to which Dr. W. H. Mills devoted his presidential address, namely, the value of the tetrahedral atom model when expressed in terms of the electronic theory of valency. The three dimensional extension of the octet theory can be simply represented in a diagrammatic manner by placing the four pairs of electrons at the corners of a tetrahedron concentric with the atom under consideration. Another atom, linked by a pair of electrons, will be situated on the axis through the corresponding axis of the tetrahedron. This tetrahedral octet can only be expected to give a general indication of configuration; since atoms are deformable and valencies can be deflected, it cannot be used, for example, to predict accurate values of intervalency angles. Qualitatively, however, it is able to represent the stereochemistry of all compounds formed in accordance with the octet rule.

When such compounds contain only links composed of two electrons, then it is invariably found that a four co-ordinate atom has a tetrahedral configuration, a three co-ordinate atom a pyramidal configuration, and a two co-ordinate atom an angular configuration. This is illustrated by the configurations of methane, ammonia and water in Fig. 1 .
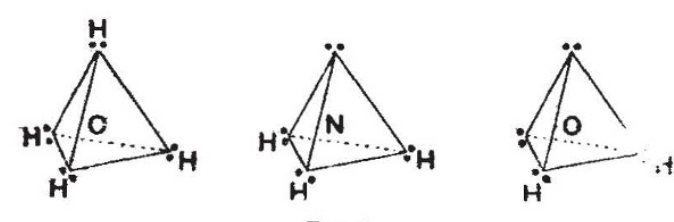

Fra. 1.

The tetrahedral type covers not only the familiar carbon compounds but also accounts for the optical aetivity of substituted ammonium ions of the type $[N a b c d]^{+}$, of amine oxides, and of four co-ordinate complexes of beryllium, copper and zinc. The pyramidal formula is, of course, in harmony with the structure of ammonia deduced from its dipole moment and infra-red spectrum ; it also accounts naturally for the isomerism of the oximes, the optical activity of sulphoxides and sulphinic esters observed by Kenyon and Phillips, and for the recent resolutions of the aci-forms of secondary nitroparaffins. The latter are of particular interest since they probably contain a tri-covalent carbon atom with a pyramidal configuration. (Fig. 2.)

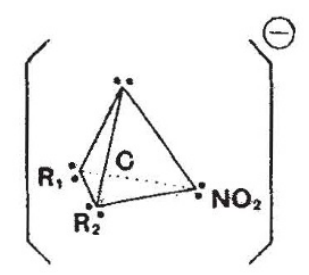

Fro. 2.

The outstanding difficulty in this group of compounds is the persistent failure to resolve amines of the type Nabc. Prof. J. Meisenheimer's contribution to the discussion was concerned mainly with this point; he showed that no resolution is obtained with compounds in which one of the nitrogen valencies is attached to oxygen, namely, in hydroxylamines and in quinoline oxide. Negative evidence of this kind may, as Sir William Pope remarked, have little or no significance and must be very cautiously interpreted ; on the other hand, there is some spectroscopic evidence for the view that the nitrogen atom oscillates through the plane containing the attached groups and so makes it impossible to isolate an optically active product.

The idea of the movement of a critical atom to a new permanent position in the molecule was shown by Dr. Mills to give a simple and natural interpretation of three important stereochemical problems, namely, the Walden inversion, the trans elimination of water from aldoximes, and the trans Beckmann change. The mechanism by which the first of these changes is brought about can be represented very simply by the formulæ in Fig. 3 .
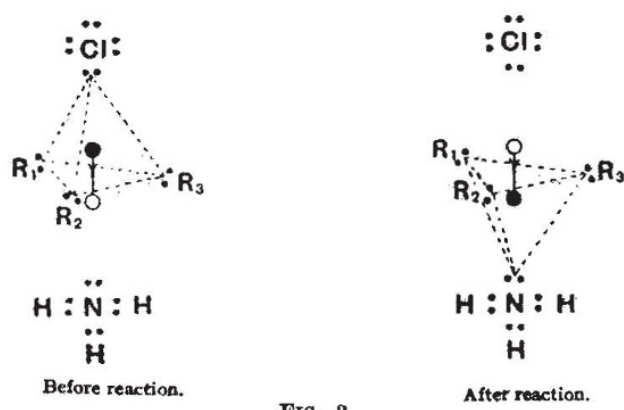

FIA. 3.

The reaction considered here is the action of ammonia on a compound containing chlorine, which results in the liberation of chlorine as an anion and the formation of a substituted ammonium ion with an inverted configuration. This result is naturally and simply represented by the movement of the asymmetric carbon atom through a distance equal to half the height of the tetrahedron. The critical point in this explanation is, of course, the attack of the ammonia molecule on

No. 3285, VoL. 130] 
the correct face of the tetrahedron, since attack at any other face would not give the inverted configuration. The electrostatic fields due to the dipole moments of the $\mathrm{C}-\mathrm{Cl}$ link and the $\mathrm{N}-\mathrm{H}$ link will give a directing effect and the movement of the carbon atom in this direction will most readily facilitate the escape of the chlorine ion. Thus the velocity of reaction at the proper face will be favoured and inversion will be almost complete.

The formation of benzonitrile from the $\beta$-form of benzaldoxime acetate is represented in Fig. 4 . The essential reaction is the removal of the elements of acetic acid; this is brought about readily by the action of alkalis on the $\beta$-form in
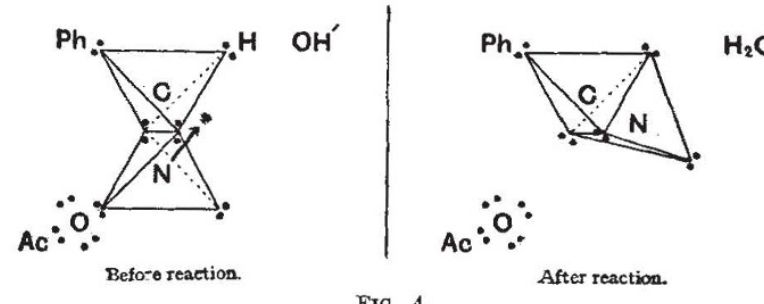

Fia. 4.

which the acetyl group and the hydrogen are on opposite sides of the molecule. The first step is the removal of a proton by union with a hydroxyl ion. The second is the movement of the nitrogen atom into line with the carbon atom to form the linear grouping of benzonitrile. This movement is directly away from the acetate group, which is, therefore, liberated as an ion. The movement of the nitrogen atom in the acetate of the isomeric $\alpha$-oxime would not be in a direction away from the acetate group; hence the isomeride which reacts most readily must have the trans configuration in accordance with the modern view.

This conception of the movement of critical atoms gives a particularly neat explanation of the trans Beckmann change. It is usual to think of the migration of groups. If, however, we consider the change occurring in an isolated molecule and remember that moment of momentum must be conserved, it is clear that most of the movement by which the relative displacement is brought about would be executed by the nitrogen atom. Fig. 5 indicates the alternative movements of the nitrogen atoms corresponding with cis- and transmigration, and show that the latter is much more probable. The driving force which brings about the change is probably the affinity of the oxygen for the central carbon atom. The first step would therefore be the attachment of the oxygen atom to this carbon atom, followed, or accompanied by the migration of the nitrogen atom. As the diagram shows, this could readily occur to give trans-migration, whilst cis-migration would involve the passage of the nitrogen atom across the line of closest approach of the oxygen atom to the central carbon atom. The cis-interchange of groups, instead of being an assumption which could be taken for granted, is a highly improbable hypothesis and the natural course of the reaction is that leading to trans-interchange.

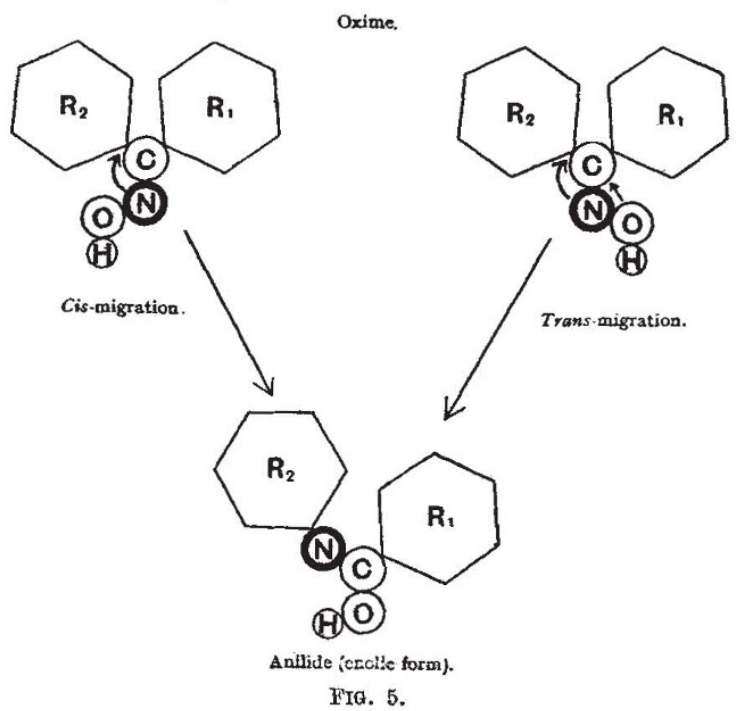

The second part of Dr. Mills's address was concerned with the problem of the optical activity of living matter. Pasteur was inclined to ascribe this to cosmical causes and later workers have invoked the influence of circularly polarised light. Dr. Mills suggested, however, that the production of dissymmetric products by living tissue is intimately connected with the characteristic property of growth which differentiates living matter from dead.

The synthesis of dissymmetric products in living tissue involves, at least in its later stages, reactions between dissymmetric substances. Such reactions are known to be stereo-specific and the velocity of the reaction is much greater with one of the optical forms than with the other. This stereospecificity is observed in comparatively simple compounds and can readily be accounted for by the shape and disposition of the parts of the molecule; in the favoured reaction the groups which react can more readily be brought into juxtaposition. With enzyme reactions a high degree of specificity is found and these reactions undoubtedly play an important part in the growth of living tissue.

From a detailed analysis of simplified models of growing tissue, Dr. Mills concludes that the optical activity of living matter is an inevitable consequence of its property of growth. Owing to the stereospecificity of the later changes, any small lack of balance between the $d$ and $l$ forms of the first dissymmetric product in the chain of reactions would rapidly increase and the dissymmetric tissue would grow more rapidly and soon be present in the growing organism in overwhelming amount. "The mystery of living matter seems to lie in its power of growth. Given this, the optical activity of its components appears to follow as a necessary consequence of the law of mass action and the stereospecificity of interactions between dissymmetric compounds." 